\title{
TOWARDS A NON-PERTURBATIVE RENORMALIZATION OF EUCLIDEAN QUANTUM GRAVITY
}

\author{
Z. Burda ${ }^{a}$ ], J.-P. Kownacki ${ }^{b}$ and A. Krzywicki ${ }^{b}$ \\ ${ }^{a}$ Fakultät für Physik, Universität Bielefeld, Bielefeld 33501, Germany \\ ${ }^{b}$ LPTHE, Bât 211, Université de Paris-Sud, 91405 Orsay, Francę
}

\begin{abstract}
A real space renormalization group technique, based on the hierarchical baby-universe structure of a typical dynamically triangulated manifold, is used to study scaling properties of $2 \mathrm{~d}$ and $4 \mathrm{~d}$ lattice quantum gravity. In $4 \mathrm{~d}$, the $\beta$-function is defined and calculated numerically. An evidence for the existence of an ultraviolet stable fixed point of the theory is presented.
\end{abstract}

May 1995

LPTHE Orsay 95/34

\footnotetext{
${ }^{1}$ Permanent address: Institute of Physics, Jagellonian University, ul. Reymonta 4, PL-30 059, Kraków, Poland

${ }^{2}$ Laboratoire associé au CNRS, URA-D0063
} 
1. As is well known, the renormalization group (RG) is a tool providing deep insight into the structure of a quantum field theory. It is certainly worth applying this tool to quantum gravity. This work is devoted to the development and the application of the real space renormalization group technique in the context of euclidean quantum gravity. It is a direct continuation of the work [1].

We choose the lattice gravity framework. More precisely we adopt the particularly promising dynamical triangulation approach [2]. The remarkable results obtained within a class of exactly solvable models in two dimensions strongly suggest that the dynamical triangulation recipe is the correct way of discretizing gravity (at least for fixed topology).

In conventional statistical mechanics a real space renormalization group transformation has two facets:

(a) geometry - cells of the body are "blocked" together.

(b) matter fields - "block" fields are defined in terms of the original fields.

On a regular lattice it is trivial to perform the step (a) in such a manner that the resulting lattice is identical, modulo rescaling, to the original one. Since the values of critical couplings depend on the lattice type this selfsimilarity feature of the transformation is important. On a random lattice an appropriate definition of (a) requires some thought. In this work we consider pure geometry, without matter fields, and consequently the geometrical aspect of the renormalization group.

In ref. [1] a method of "blocking" triangulations that exploits the selfsimilarity feature of random manifolds has been proposed. Without repeating in detail the arguments presented in [1] let us briefly sketch the main idea [3. The intuitive arguments given below will be replaced progressively by more precise ones later on. We do not wish to give an impression of complexity from the outset.

2. In $2 \mathrm{~d}$ one can show [4] that an infinite randomly triangulated manifold is a self-similar tree obtained by gluing together sub-structures called baby universes (BUs), defined as subuniverses separated from the remaining part of the universe by a narrow neck. We conjecture that a similar picture holds in $4 \mathrm{~d}$, at least in the neighborhood of the phase transition point. The results presented in this paper strongly support this conjecture.

\footnotetext{
${ }^{3}$ As we have learned from J. Ambjørn during the LATTICE '94 Conference, our ideas partly overlap with those discussed earlier for $2 \mathrm{~d}$ in ref. [3].
} 
We have proposed [1] to define the step (a) as the operation of cutting the last generation of BUs, i.e. those which have no further BUs growing on them. When the tree is finite, and as one continues cutting the successive generations of BUs, it gets smaller (in lattice units) and looks less and less branched. In the spirit of the renormalization group this is interpreted as reflecting the reduction of the resolving power: although, in physical units, the manifold remains of the same size, the fine structure is not observed.

The next problem consists in establishing a connection between lattice and physical observables. For example, in lattice QCD one identifies the inverse of the correlation length $\xi$ with the mass $m$ of a hadron

$$
m a=\xi^{-1}
$$

Here $a$ denotes the lattice spacing and $\xi=\xi(\kappa)$ depends on a dimensionless coupling constant $\kappa$. The correlation length $\xi$ can be calculated for a given $\kappa$. The mass $m$ is a physical observable, independent of the regularization procedure. Hence, eq. (11) yields the variation of the lattice spacing $a$, measured in physical units, with the coupling $\kappa$. The so-called $\beta$-function can by defined as

$$
\beta(\kappa)=\frac{d \kappa}{d \ln (1 / a)},
$$

where the derivative is taken along the RG flow. In QCD, the lattice correlation length diverges exponentially as $\kappa \rightarrow 0: \xi \propto \exp \left(1 / 2 \beta_{0} \kappa^{2}\right)$ with $\beta_{0}>0$. Eq. (11) implies that $\beta(\kappa)=-\beta_{0} \kappa^{3}$. The fixed point is at $\kappa=0$ and is ultraviolet (UV) stable. The lattice spacing tends to zero, in physical units set by eq. (1), as one approaches the fixed point.

We recall these very well known things, in a sense, to set the standards. We would like to have similar arguments in quantum gravity. The phase transition observed in 4d [5, 6] seems to be continuous and therefore one has a candidate for a fixed point. The nature of this fixed point (IR or UV) is to be found. The soundness of the proposed renormalization group has to be further examined. Finally, one has to select appropriate observables in order to fix the scales. We shall report about some progress achieved in studying these problems. But before proceeding we have to define the theory we are working with.

4. We take an euclidean version of the Einstein-Hilbert action which for a simplicial manifold reads

$$
S=-\kappa_{2} N_{2}+\kappa_{4} N_{4}
$$


where $N_{2}$ and $N_{4}$ denote the total number of triangles and 4-simplexes, respectively. The theory is defined by the partition function

$$
Z\left(\kappa_{2}, \kappa_{4}\right)=\sum_{N_{2}, N_{4}} Z_{N_{2} N_{4}} e^{-S}
$$

where

$$
Z_{N_{2} N_{4}}=\sum_{T\left(N_{2}, N_{4}\right)} W(T)
$$

The sum in eq. (5) runs over fixed topology $4 \mathrm{~d}$ simplicial manifolds $T\left(N_{2}, N_{4}\right)$ with $N_{2}$ triangles and $N_{4}$ 4-simplexes. $W(T)$ is the symmetry factor taking care of the equivalent relabelings of the manifold.

We study a fixed volume $N_{4}=N$ canonical ensemble for spherical manifolds with the partition function :

$$
Z\left(\kappa_{2}, N_{4}\right)=\sum_{N_{2}} Z_{N_{2} N_{4}} e^{-S}
$$

In practice, in order to have an ergodic algorithm, one is forced to simulate a multicanonical ensemble (see e.g. [7]). One lets the volume fluctuate in an external potential $U\left(N_{4}\right)$ with parameters adjusted in such a way that the entry $N_{4}=N$, at which measurements are done, is highly probable in the resulting $N_{4}$ distribution. In our simulations we have used $U=\frac{\delta}{2}\left(N-N_{4}\right)^{2}$ with $\delta=0.001$.

The observables have to be geometrical objects. One obvious choice is the total physical volume $V=N a^{4}$. Once $V$ has been fixed, the length of the lattice step in physical units is also fixed for a given lattice:

$$
a=(V / N)^{\frac{1}{4}}
$$

However, the continuum limit of the theory is not yet defined. A second observable is needed for that. A priori, this second observable could be the local curvature $R$. It is, however, not quite obvious how the physical curvature should actually be defined. In numerical simulations of dynamically triangulated $4 \mathrm{~d}$ manifolds, the bare Regge curvature residing on the hinges of the lattice is found to be nonvanishing at the transition point [5, 6, 8], which leads to basic problems in defining its dimensionful counterpart in the continuum limit. Various interpretations have been given to this finding. We are tempted to adopt here the point of view formulated by the authors of ref. 
[9], who argue in substance that the bare curvature is a lattice artifact and is not the curvature that would measure an observer using a physical stick, independent of the cut-off.

Following the suggestion put forward in [9], we choose as our second observable a length scale characterizing the large-distance behavior of the so-called puncture-puncture correlations function, viz. the average geodesic distance between two randomly selected simplexes of the manifold:

$$
\langle r\rangle=\left\langle N^{-2} \sum_{a b} r_{a b}\right\rangle_{N}
$$

where $r_{a b}$ is the geodesic distance between 4 -simplexes $a$ and $b$ and $\langle\ldots\rangle_{N}$ denotes the average over manifolds described by the canonical partition function (6).

In practice, it is difficult to identify BUs with necks larger than the minimum one. Following the terminology introduced in ref. [四] we call minBUs the baby universes with the smallest possible neck. We have sugested in [1] that a meaningful RG transformation is defined by the operation of cutting last generation minBUs. Using the pure $2 \mathrm{~d}$ quantum gravity as a toy model, we have checked that, on the average, the area of surfaces obtained after cutting last generation minBUs is, for large surfaces, a constant fraction of the area of the original surface. It is important to check that a similar result holds for $\langle r\rangle$, if $\langle r\rangle$ is to be used in a RG analysis.

5. We have been working with surfaces of area ranging between $N=4096$ and 65536 triangles. The minimum number of triangles in a minBU has been set to 15. The number of heating sweeps between measures has been set to twice the autocorrelation length determined, for the observable $\langle r\rangle$, in ref. [10] 1. The results are shown in Table 1. The subscripts "in" and "out" refer to the original and the cut surfaces, respectively. Actually, the size of the original surface has been kept fixed : $N_{\text {in }} \equiv N$.

It appears from the results presented in the Table 1 that the ratios $\lambda_{N}=$ $\left\langle N_{\text {out }}\right\rangle / N_{\text {in }}$ and $\lambda_{r}=\left\langle r_{\text {out }}\right\rangle /\left\langle r_{\text {in }}\right\rangle$ converge towards limiting values when $N \rightarrow$ $\infty$. Furthermore, the critical exponent $\nu$ defined by the relation $\lambda_{r}=\lambda_{N}^{\nu}$ approaches the expected value $\frac{1}{4}$. Let us recall that the problem of scaling in

\footnotetext{
${ }^{4}$ Strictly speaking, they have done this for a model with $2 \mathrm{~d}$ gravity coupled to a gaussian field; it does not seem that autocorrelation lengths are longer for pure gravity when one measures such a purely geometrical object as the average geodesic distance.
} 
pure $2 \mathrm{~d}$ gravity has been solved rigorously $[11]$ and it is known that $\langle r\rangle \propto N^{\frac{1}{4}}$ as $N \rightarrow \infty$. This could have been checked numerically with better precision using a more direct method (see e.g. ref. [12]). Our point is that $\langle r\rangle$ scales correctly under the RG transformation we have proposed. Notice, that selfsimilarity and scaling only hold in the statistical sense: it is the grandcanonical ensemble that is mapped into itself by our transformation.

In $2 \mathrm{~d}$ gravity one can write for small enough $a$

$$
\langle r\rangle=(\rho / a)^{2 \nu}\left(\nu=\frac{1}{4}\right)
$$

where $\rho$ is an observable with the dimension of a length, which indirectly determines the physical curvature of the surface. Eq. (9) is an analogue of eq. (目) and $\rho$ could be used to set the scale of the theory. However, once the physical area $V=N a^{2}$ has been used for that purpose, the choice of $\rho$ is no longer free.

Playing with the toy model we have convinced ourselves that $\langle r\rangle$ is an observable suitable for our purposes. We also got better acquainted with the proposed RG transformation. Thus, when one cuts the successive generations of minBUs, the corresponding scaling factors $\lambda_{N}^{(1)}, \lambda_{N}^{(2)}, \ldots, \lambda_{N}^{(n)}, \ldots$ are not at all equal and actually rapidly tend to unity. This is easily understood from the results of ref. [4]: before the outer minBUs have been cut, the minBUs of the next layer are obviously more volumineous than the outer ones (since the latter are just outgrows off the former). In order to keep $\lambda_{N}$ constant, one should enlarge the necks of BUs to be cut at each iteration of the RG transformation. This is not what we are doing, since we restrict ourselves, for purely technical reasons, to minBUs.

The results shown in Table 1 have been obtained iterating the transformation until no minBU is left. When only one iteration is performed, the value of $\lambda_{N}$ is larger (about 0.86 , with the same definition of a minBU) and $\lambda_{r}$ is very close to unity. Consequently, the error in the determination of $\nu$ is much larger, for the same statistics, and the results appear less convincing.

6. Encouraged by the results obtained in $2 \mathrm{~d}$ we go over to $4 \mathrm{~d}$. Now, $\langle r\rangle$ is a function of two variables, viz. $N$ and $\kappa_{2}$, to be denoted $\kappa$ from here on for the sake of simplicity of writing.

Suppose we start with $N=N_{i n}$ and $\kappa=\kappa_{\text {in }}$ and calculate the corresponding average geodesic distance $\langle r\rangle=\left\langle r_{i n}\right\rangle$. After one iteration of RG we find $\left\langle N_{\text {out }}\right\rangle$ and $\left\langle r_{\text {out }}\right\rangle$. Denote by $\kappa_{\text {out }}$ the value of $\kappa$ characterizing the new 
ensemble. Define

$$
\begin{aligned}
\delta r & =\left\langle r_{\text {in }}\right\rangle-\left\langle r_{\text {out }}\right\rangle \\
\delta N & =N_{\text {in }}-\left\langle N_{\text {out }}\right\rangle \\
\delta \kappa & =\kappa_{\text {in }}-\kappa_{\text {out }}
\end{aligned}
$$

From (7) we get

$$
\delta \ln (1 / a)=\frac{1}{4} \delta \ln N
$$

The associated shift of $\kappa$ can be found from the equation

$$
\delta r=r_{N} \delta \ln N+r_{\kappa} \delta \kappa
$$

where $r_{N}$ and $r_{\kappa}$ are the partial derivatives of $\langle r\rangle$ with respect to $\ln N$ and $\kappa$, which can also be estimated from numerical simulations. Once $\delta \kappa$ has been calculated, the $\beta$-function is readily found from (2) and (13). Writing eq. (15) we have actually conjectured that $\kappa$ is the only relevant coupling at long distances. This is presumably reasonable when $\kappa$ is close enough to its critical value. One can check the conjecture by calculating the $\beta$-function using some other observable than $\langle r\rangle$, to fix the scale. If the conjecture is true the result should not depend appreciably on the choice of the observable.

We have applied our RG transformation to 4d spherical simplicial complexes with 8000 simplexes, working with a series of values of $\kappa$ ranging between 0.9 and 1.45 . The average geodesic distance has also been calculated, for the same values of $\kappa$ at $N=6000$, in order to estimate $r_{N}$. The five standard local moves have been used for updating. Typically, we have been carrying $\sim 10^{5}$ sweeps of the lattice, for each $\kappa$. Close to the critical point the number of sweeps has been significantly larger. Measures were separated by a number of heating sweeps, equal to twice the autocorrelation length, estimated in advance. The errors were found using the conventional binning method. The minimum volume of a minBU has been set to 20 simplexes. The RG transformation has been iterated only once. Above $\kappa=1.15$ cutting more than one generation of minBUs yields too small $\left\langle N_{\text {out }}\right\rangle$ for the differential formula (14) to be trusted The data are collected in Table 2 and the

\footnotetext{
${ }^{5}$ At $\kappa=1.3$, iterating RG the maximum number of times one finds $\left\langle N_{\text {out }}\right\rangle=859 \pm 300$ :
} 
$\beta$-function is shown in Fig. 1. A linear fit to the data yields $\beta(\kappa)=\beta_{0}\left(\kappa^{*}-\kappa\right)$ with $\beta_{0}=6.5(1.2)$ and $\kappa^{*}=1.2(2)$.

Although the errors are large, especially as one enters the branched polymer phase, where the local moves are not very efficient, it is rather evident from Fig. 1 that the critical point is an ultraviolet stable one ${ }^{6}$. Notice, that a qualitatively identical result has been found analytically in $2+\epsilon$ dimensions [13, 14], using perturbation theory together with the $\epsilon$ expansion. Let us now briefly outline some general consequences of this result.

7. Close enough to the fixed point $\kappa=\kappa^{*}$ one can write

$$
\frac{d \kappa}{d \ln (1 / a)}=\beta_{0}\left(\kappa^{*}-\kappa\right)\left(\beta_{0}>0\right)
$$

Solving this differential equation one finds

$$
a=a_{0}\left|\kappa^{*}-\kappa\right|^{1 / \beta_{0}} \quad\left(\beta_{0}>0\right)
$$

where $a_{0}$ is an integration constant, analogous to $\Lambda^{-1}$ in QCD, and which should be given a value, in physical units, in order to define the theory. Comparing with (7) one gets

$$
V / a_{0}^{4}=t
$$

with $t$ defined by

$$
t=N\left|\kappa^{*}-\kappa\right|^{4 / \beta_{0}}
$$

The quantity on the left-hand side of (17) is a constant, determined by the choice of $V$ and $a_{0}$. The trajectories of the RG flow are the curves $t=$ const. The continuum limit is the double-scaling limit: $N \rightarrow \infty, \kappa \rightarrow \kappa^{*}$ and $t=$ const. In analogy to $2 \mathrm{~d}$ one can write $\langle r\rangle=(\rho / a)^{4 \nu}$, but now $\rho$ and $\nu$ may depend on the value given to $t$.

8. Going farther ahead is beyond the scope of this paper. A few remarks are in order at this point :

(i) This study should and will be extended to larger lattices. In order to do that it will be necessary to use a more efficient updating algorithm. Indeed,

the manifolds resemble branched polymers and the situation differs from that described in sect. 5 .

${ }^{6} \mathrm{P}$. Bialas has informed us that using the data of ref [9] and our definition of the $\beta$-function one can estimate the latter in 3 points. The estimates agree with our result. 
the local algorithm becomes inefficient as one enters the "cold" phase of the theory, where the manifold develops a branched polymer structure. The "BU surgery" algorithm proposed in [10, 15] should be appropriate there. Performing more intensive $M C$ studies one should also be able to estimate the corrections to the formula (14) coming from irrelevant directions at the fixed point, neglected here.

(ii) Remember, that $\nu^{-1}$ has the significance of the intrinsic Haussdorf dimension $d_{H}$ of the manifold [15]. It would be interesting to look for the $t$ dependence of $d_{H}$ and determine the class of theories satisfying the constraint $d_{H}=4$. Our experience with $2 \mathrm{~d}$ indicates that this may require going to very large systems.

(iii) It would be very interesting to extend the analysis to models where matter fields are coupled to gravity and to achieve a better understanding of the relation between the results generated by lattice models and the continuum calculations in $2+\epsilon$ dimensions. With a more efficient algorithm and a better statistics it will hopefully be possible to examine the dependence of the shape of the $\beta$-function on the number of matter fields. Calculations in the continuum [13, 14] suggest something analogous to the $c=1$ barrier of $2 \mathrm{~d}$ models: the number of matter fields cannot be arbitrary for the theory to exist. It would be important to obtain such a result in lattice gravity, without any recourse to perturbation theory. It might be that this has some relevance for the problem of the number of generations.

In summary, we have applied the previously proposed real space renormalization group procedure to $2 \mathrm{~d}$ and $4 \mathrm{~d}$ models of pure euclidean quantum gravity. In $4 \mathrm{~d}$ we have defined a $\beta$-function and we have found numerically some evidence for the existence of an ultraviolet stable fixed point of the theory. We have concluded mentioning a few problems for the future.

Acknowledgements : We have benefited from discussions and exchange of information with P. Bialas, D. Johnston, J. Jurkiewicz and B. Petersson. In particular, J. Jurkiewicz has called our attention to ref. [9] and to the potential interest of the distribution of geodesic distances. The help of B. Klosowicz has been invaluable. We are indebted to the CNRS computing center IDRIS, and personally to V. Alessandrini, for computer time and cooperation. One of us (Z.B.) is grateful to Deutsche Forschungsgemeinschaft for financial support. 


\section{References}

[1] D. Johnston, J.-P. Kownacki and A. Krzywicki, in LATTICE '94, Nucl. Phys. B (Proc. Suppl.)42 (1995) 728.

[2] D. Weingarten, Nucl. Phys. B210 [FS 6] (1982) 229; F. David, Nucl.Phys. B257 (1985) 543 ; J. Ambjørn, B. Durhuus and J. Fröhlich, Nucl. Phys. B257 (1985) ; V.A. Kazakov, I.K. Kostov and A.A. Migdal, Phys. Lett. B157 (1985) 295.

[3] J. Ambjørn, B. Durhuus, J. Fröhlich and T. Jonsson, J. Stat. Phys. 55 (1989) 29.

[4] S. Jain and S.D. Mathur, Phys. Lett. B286 (1992) 239.

[5] J. Ambjørn and J. Jurkiewicz, Phys. Lett.B278 (1992) 50.

[6] M.E. Agishtein and A.A. Migdal, Mod. Phys. Lett. A7 (1992) 1039; Nucl. Phys. B385 (1992) 395.

[7] S. Bilke, Z. Burda and J. Jurkiewicz, Comp. Phys. Comm. 85 (1995) 278.

[8] J. Ambjørn, J. Jurkiewicz and C.F.Kristjansen, Nucl. Phys. B393 (1993) 601.

[9] B.V. De Bakker and J. Smit,Nucl. Phys. B439 (1995) 239.

[10] J. Ambjørn, P. Bialas, Z. Burda, J. Jurkiewicz and B. Petersson, Phys. Lett. B325 (1995) 337.

[11] H. Kawai,N. Kawamoto, T. Mogami and Y. Watabiki, Phys. Lett. B306 (1993) 19 ; see also J. Ambjørn and Y. Watabiki, Scaling in quantum gravity, Copenhagen preprint NBI-HE-95-01 (January 1995) and references therein.

[12] S. Catterall, G. Thorleifsson, M. Bowick and V. John, Syracuse preprint SU-4240-607 (April 1995). 
[13] S. Weinberg, in General Relativity, an Einstein Centenary Survey, eds. S.W. Hawking and W. Israel (Cambridge University Press 1979); R. Gastmans, R. Kallosh and C. Truffin, Nucl. Phys. B133 (1978) 417; S.M. Christensen and M.J. Duff, Phys. Let. B79 (1978) 213; more recently the problem has been thoroughly discussed in a series of papers of the Japanese group [14].

[14] H. Kawai and M. Ninomiya, Nucl. Phys. B336 (1990) 115 ; H. Kawai, Y. Kitazawa and M. Ninomiya, Nucl. Phys. B404 (1993) 280; ibid B427 (1993) 684 ; T. Aida, Y. Kitazawa, H. Kawai and M. Ninomiya, Nucl. Phys. B427 (1994) 158 ; T. Aida, Y. Kitazawa, J. Nishimura and A. Tsuchiya, Tokyo preprint TIT-HEP-275 (December 1994).

[15] J. Ambjørn and J. Jurkiewicz, Scaling in four dimensional quantum gravity, Copenhagen preprint NBI-HE-95-05 (February 1995).

\section{Figure caption}

Fig. 1 - The $\beta$-function versus the coupling $\kappa$ in 4 d, calculated for spherical manifolds with 8000 simplexes. 


\begin{tabular}{|c|c|c|c|c|}
\hline$N$ & $\left\langle r_{\text {in }}\right\rangle$ & $\frac{\left\langle r_{\text {out }}\right\rangle}{\left\langle\mathrm{r}_{\text {in }}\right\rangle} \equiv \lambda_{r}$ & $\frac{\left\langle N_{\text {out }}\right\rangle}{N} \equiv \lambda_{\mathrm{N}}$ & $\begin{array}{c}\nu \text { from } \\
\lambda_{r}=\lambda_{\mathrm{N}}^{\nu}\end{array}$ \\
\hline 4096 & $31.20(22)$ & $0.880(8)$ & $0.745(6)$ & $0.433(33)$ \\
8192 & $39.34(24)$ & $0.900(5)$ & $0.752(7)$ & $0.371(24)$ \\
16384 & $48.99(33)$ & $0.919(8)$ & $0.764(5)$ & $0.312(37)$ \\
32768 & $61.29(63)$ & $0.928(13)$ & $0.763(4)$ & $0.276(50)$ \\
65536 & $75.12(98)$ & $0.935(16)$ & $0.762(3)$ & $0.247(63)$ \\
\hline$\infty$ & $\infty$ & $0.935^{(c)}$ & $0.765^{(b)}$ & $0.25^{(a)}$ \\
\hline
\end{tabular}

(a) Theoretical result from ref. [11].

(b) Obtained by linearly extrapolating data.

(c) Obtained from (a) and (b) using $\lambda_{r}=\lambda_{N}^{\nu}$

Table 1: Summary of data in 2d.

\begin{tabular}{|c||c|c|c||c|}
\hline \multicolumn{1}{|c||}{} & \multicolumn{3}{c||}{$N=8000$} & $N=6000$ \\
\hline$\kappa_{2}$ & $\left\langle r_{\text {in }}\right\rangle$ & $\left\langle r_{\text {out }}\right\rangle$ & $\left\langle N_{\text {out }}\right\rangle$ & $\left\langle r_{\text {in }}\right\rangle$ \\
\hline 0.9 & $11.907(26)$ & $11.538(25)$ & $7422(19)$ & - \\
1.0 & $12.661(36)$ & $11.97(10)$ & $7013(76)$ & $12.191(10)$ \\
1.1 & $14.28(14)$ & $13.20(12)$ & $6586(118)$ & $13.804(41)$ \\
1.15 & $15.80(21)$ & $14.58(30)$ & $6464(203)$ & $16.01(33)$ \\
1.2 & $23.27(95)$ & $21.67(95)$ & $6098(143)$ & $22.18(39)$ \\
1.25 & $31.10(60)$ & $29.41(60)$ & $6101(132)$ & $27.89(61)$ \\
1.3 & $34.06(73)$ & $32.35(85)$ & $6035(138)$ & $28.93(69)$ \\
1.4 & $36.77(80)$ & $35.01(85)$ & $5968(160)$ & $32.05(66)$ \\
1.45 & $37.55(84)$ & $35.83(87)$ & $5980(347)$ & - \\
\hline
\end{tabular}

Table 2: Summary of data in $4 \mathrm{~d}$. 
FIGURE 1

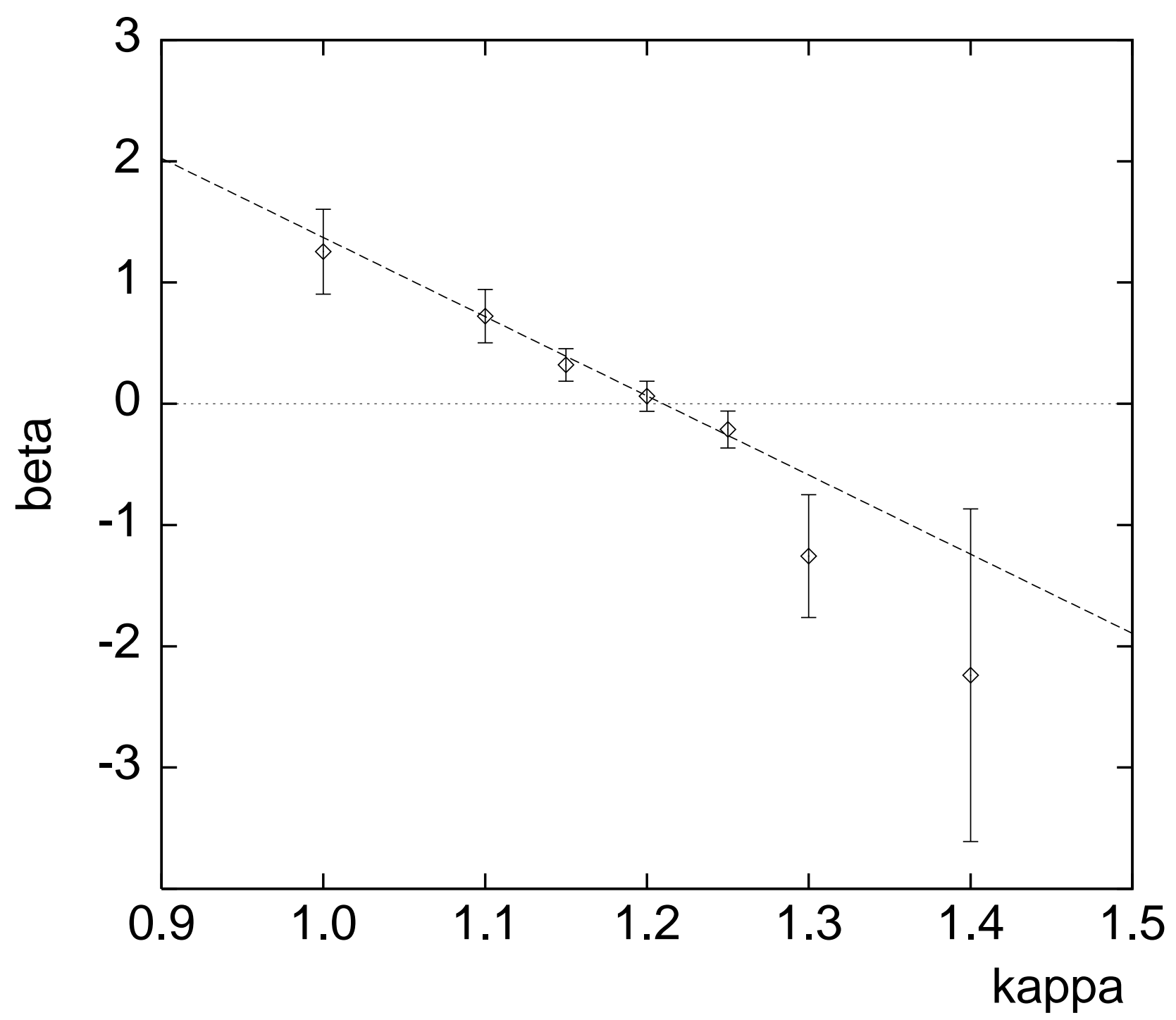

